\title{
Article
}

\section{Orbital symmetry matching: Achieving superior nitrogen reduction reaction over single-atom catalysts anchored on Mxene substrates}

\author{
Jiale Qu †, Jiewen Xiao †, Hetian Chen, Xiaopeng Liu, Tianshuai Wang, Qianfan Zhang* \\ School of Materials Science and Engineering, Beihang University, Beijing 100191, China
}

\section{A R T I C L E I N F}

\section{Article history:}

Received 17 March 2020

Accepted 30 April 2020

Published 5 February 2021

\section{Keywords:}

Orbital symmetry matching

Single atom catalysis

Nitrogen reduction reaction

MXene substrate

Potential determining step

\begin{abstract}
A B S T R A C T
The nitrogen reduction reaction (NRR) under ambient conditions is still challenging due to the inertness of $\mathrm{N}_{2}$. Herein, we report a series of superior NRR catalysts identified by examining $\mathrm{Ti}_{2} \mathrm{NO}_{2}$ MXenes embedded with 28 different single-atom catalysts using first-principles calculations. The stability of this system was first verified using formation energies, and it is discovered that $\mathrm{N}_{2}$ can be effectively adsorbed due to the synergistic effect between single atom catalysis and the Ti atoms. Examination of the electronic structure demonstrated that this design satisfies orbital symmetry matching where "acceptor-donor" interaction scenario can be realized. A new "enzymatic-distal" reaction mechanism that is a mixture of the enzymatic and distal pathways was also discovered. Among all of the candidates, $\mathrm{Ni}$ anchored on MXene system achieves an onset potential as low as $-0.13 \mathrm{~V}$, which to the best of our knowledge is the lowest onset potential value reported to date. This work elucidates the significance of orbital symmetry matching and provides theoretical guidance for future studies.
\end{abstract}

(C) 2021, Dalian Institute of Chemical Physics, Chinese Academy of Sciences. Published by Elsevier B.V. All rights reserved.

\section{Introduction}

As an ingredient that can be directly absorbed by plants for the biosynthesis of basic blocks of life, ammonia plays an essential role in the fertilizer industry and in the production of nitrogen-containing compounds [1,2]. Traditionally, industrial production of $\mathrm{NH}_{3}$ is carried out using the Haber-Bosch process [3-5] that requires both high pressure and temperature to promote the dissociation of nitrogen. This approach not only leads to high energy consumption [6], but is also restricted by the Brønsted-Evans-Polanyi (BEP) relation that ensures that a lower $\mathrm{N}_{2}$ dissociation barrier will result in the higher $\mathrm{NH}_{x}$ desorption impedance $[7,8]$, limiting the optimum performance of nitrogen reduction reaction (NRR) catalysis. Therefore, biological fixation of $\mathrm{N}_{2}$ using nitrogenase enzymes has been exten- sively studied, and the electrochemical $\mathrm{N}_{2}$ reduction reaction (eNRR) was also proposed [9-12]. In the eNRR process, the production efficiency can be promoted by controlling the operating potential, $\mathrm{pH}$, and electrolytes [13]. Among the various strategies for increasing eNRR efficiency, catalyst design is the most effective and intrinsic strategy, and various transition metal (TM)-based catalysts have been proposed such as the transition metal-dinitrogen complex with a PNP-pincer [14,15], Mo embedded in BN [16], and transition metal (TM)-doped carbon-based materials [17]. However, most of these catalysts show a large overpotential toward NRR and exhibit low Faradaic efficiency [18-21].

Recently, single-atom catalysis (SAC) has received considerable attention and has emerged as a new frontier in the field of heterogeneous catalysis [22]. In an SAC system, active iso-

\footnotetext{
* Corresponding author. Tel: +86-15210659892; Fax: +86-10-82314478; E-mail: qianfan@buaa.edu.cn

†These authors contributed equally to this work.

This work was supported by National Natural Science Foundation of China (11404017).

DOI: 10.1016/S1872-2067(20)63643-9 | http://www.sciencedirect.com/science/journal/18722067 | Chin. J. Catal., Vol. 42, No. 2, February 2021
} 
lated metal atoms can be highly dispersed in the supporting framework; this approach not only achieves superior catalytic activity, but can also significantly reduce the content of active materials [23]. Since the first work on single Pt atom catalysis toward CO oxidation [24], various TM atoms and even non-metal elements on a series of supports, including transition metal oxides [25-27], $\mathrm{N}$ doped graphene [28], $\mathrm{C}_{3} \mathrm{~N}_{4}$ [26], and $\mathrm{MoS}_{2}$ [30] have been extensively investigated. However, the aggregation of the catalyst atoms is the most formidable challenge faced by SAC, because atomic dispersion destabilizes the SAC system, inevitably creating a driving force for the aggregation of single atom catalysts to nanoparticles [31]. Therefore, the choice of the substrate plays a crucial role in SAC because the substrate must have strong interactions with the dispersed single atoms. Furthermore, suitable substrates may cooperate with SAC and further tune their electronic structures to achieve a synergetic catalytic effect. For NRR, the SAC substrates are mainly transition metal oxides [32,33] and carbon-based materials [17,34]. For instance, a single $\mathrm{Ru}$ atom supported on $\mathrm{ZrO}_{2}$ has been investigated by Tao et al. [32], and the obtained low potential of $\sim 0.17 \mathrm{~V}$ surpasses those of the other reported catalysis. Meanwhile., Liu et al. [17] have systematically screened 20 metal atoms on a series of carbon substrates but have found that the onset potential is still unsatisfactory and its optimization is hindered by the scaling relations. Therefore, the realization of excellent NRR performance depends on the choice of the substrate in the SAC design, necessitating the exploration of substrates for SAC and their effect on the eNRR performance.

MXenes represent a series of transition metal carbides and nitrides exfoliated from the MAX phase that form a family of two-dimensional materials with metallic properties [35,36]. In the field of energy storage systems, MXenes have already been applied in electrochemical supercapacitors, Li-ion and Na-ion achieving great success $[37,38]$. Additionally, the catalytic performance of MXenes has also been explored, for example for hydrogen production [39] and in the $\mathrm{CO}_{2}$ reduction reaction [40]. Moreover, Azofra et al. [41] have recently investigated the intrinsic catalytic performance of MXenes in NRR and found that it suffers from the highly active metallic surface of the MXenes. However, considering the high surface area, excellent conductivity, and stability of MXenes, their use as substrates to stabilize SAC and further modify the electronic states is quite promising. Furthermore, it is recently discovered that for $\mathrm{Ti}_{3} \mathrm{C}_{2} \mathrm{~T}_{y}$ MXene, some Ti atoms can also be etched off in the exfoliation into a monolayer, inducing Ti vacancies [42]. Such Ti deficiency can accommodate and further stabilize single-atom catalysis, as has already been realized in the functionalization process of $\mathrm{CO}_{2}$ by using single $\mathrm{Pt}$ atom catalysis [43]. Compared to the extensive investigations on carbon-based SAC substrates in the area of nitrogen fixation, the single transition metal atom catalysts anchored on MXene substrates have been relatively less explored.

In the present work, a typical $\mathrm{Ti}_{2} \mathrm{NO}_{2}$ MXene was used to accommodate 28 different single-atom-catalysis systems, and their catalytic performance toward the NRR was systematically investigated. The stability of this design is first verified using formation energy analysis and molecular dynamic simulations, and then, the $\mathrm{N}_{2}$ adsorption ability of the catalysts was examined. It was found that both side-on and end-on adsorption patterns can satisfy the orbital symmetry matching due to the synergistic effect between the SAC and the adjacent Ti atoms. Then, the electrochemical performance for NRR was investigated, and it was shown that a new enzymatic-distal mixed reaction pathway is adopted by most single-atom catalysts. Further calculations revealed that the potential determining step can be modulated based on efficient $\mathrm{N}_{2}$ adsorption. A linear scaling relation between the single $\mathrm{N}$ atom binding strength and the onset potential was established, suggesting that excellent NRR performance can be achieved by the Ni@MXene system, with the onset potential as low as $-0.13 \mathrm{~V}$. Thus, we have not only proposed a catalyst design of the single-atom catalyst anchored on the MXene substrate, but also revealed the electronic origins of its performance, providing theoretical guidance for the future studies of NRR catalysts.

\section{Computational methods}

First-principles calculations were implemented using the Vienna Ab-initio Simulation Package (VASP) in the framework of density functional theory $[44,45]$. The ion-electron interaction was described by the projector-augmented wave (PAW) pseudopotentials [46,47], and the generalized gradient approximation (GGA) parameterized according to PerdewBurke-Ernzerhof (PBE) formulation was used as the exchange-correlation functional [48]. The energy cutoff for the plane wave basis was set to $550 \mathrm{eV}$, while the Brillouin zone was sampled using a $4 \times 4 \times 1 \mathrm{k}$-mesh according to the Gamma-centered Monkhorst-Pack scheme [49]. Considering the metallic properties of the MXene substrates, the Fermi-smearing method was applied with a smearing width of 0.1 $\mathrm{eV}$. In the vertical direction, a vacuum layer was introduced to simulate a two-dimensional system. The total energy convergence threshold was set to $10^{-5} \mathrm{eV}$, while all ions were relaxed to a force tolerance of less than $0.01 \mathrm{eV} / \AA$. The Crystal Occupation Hamiltonian Population (COHP) method was applied to investigate the bonding and anti-bonding states, as implemented by LOBSTER [50-53]. Ab-initio molecular dynamics (AIMD) $[54,55]$ were applied to investigate the thermodynamic stability of the catalyst systems at room temperature $(300 \mathrm{~K})$, and the NVT ensemble was adopted with the Nose mass parameter set to 0.5 to control the temperature oscillations.

To simulate electrochemical NRR performance, we have adopted the computational hydrogen electrode model proposed by Nørskov et al. [56], for which the Gibbs free energy difference $(\Delta G)$ for each elementary step is expressed as:

$$
\Delta G=\Delta E+\Delta E_{\mathrm{ZPE}}-T \Delta S+e U
$$

where $\Delta E$ is the electronic energy difference before and after the adsorption of the reaction intermediates, $\Delta E_{\mathrm{ZPE}}$ is the difference between the zero-point energies that can be obtained from the vibrational frequency calculations. $\Delta S$ is the entropy change and $T$ is the temperature which is set to $298.15 \mathrm{~K}$. $U$ is the applied electrode potential and $e$ is the amount of the transferred charge. The lowest onset potential can be derived 
by shifting the $\Delta G_{\max }$ of the potential-determining step to zero.

\section{Results and discussion}

\section{1. $N_{2}$ adsorption and the symmetrically matched states}

Starting from the MXenes family comprised by the early transitional metals, we have adopted the $\mathrm{Ti}_{2} \mathrm{~N}$ MXene terminated by surface $\mathrm{O}$ atoms $\left(\mathrm{Ti}_{2} \mathrm{NO}_{2}\right)$ as a typical candidate substrate. Since MXenes are $d^{2}-d^{4}$ transition metal system, unlike the noble electron systems with almost a full electron shell, the few $d$ electrons generally cannot share similar orbital symmetry as that in $\mathrm{N}_{2}$, failing to effectively match the electronic states for reactants. Pure MXenes also exhibit strong binding toward nitrogen, thus poisoning catalysis [44]. Therefore, single-atom catalysis can be further introduced to tailor their electronic structures and cooperate with the metallic $\mathrm{Ti}_{2} \mathrm{NO}_{2}$ substrate as the electron reservoir. Here, we consider 28 kinds of single atoms, ranging from $3 d$ to $5 d$ metals with $d^{1}$ to $d^{10}$ occupations to screen for effective single-atom catalysts, as shown in Fig. 1(b) (radioactive elements such as Tc and lanthanide elements are not considered here). The atomic configurations of this layered system are presented in Fig. 1a, along with the general features of the partial density of states (PDOS). It is observed that the MXene substrate gives rise to two broad peaks that overlap with the electronic states of single-atom catalysts, while SAC introduces a localized state in the gap that modulates the intrinsic electronic properties of MXenes. It is observed that with the increasing number of $d$ electrons, the additional single-atom catalyst states become more localized and accordingly accommodate more $d$ electrons (Section SI), further leading to the desirable orbital symmetry matching with $\mathrm{N}_{2}$, as will be analyzed below. To demonstrate the stability of this design, formation energies ( $\left.E_{\text {form }}\right)$ have been calculated and are shown in the lower panel of Fig. 1(b), indicating that most candidate systems are thermodynamically stable. Only elements with either full or almost full electron shell such as
$\mathrm{Au}, \mathrm{Ag}, \mathrm{Zn}, \mathrm{Cd}$ and $\mathrm{Hg}$ show much smaller $E_{\text {form }}$ values. Ab-initio molecular dynamics (AIMD) simulations were further performed for these five systems and the detachment of the single-atom catalyst was observed (Fig. S6). Therefore, with the exception of these five elements, single-atom catalysts can be effectively anchored on the MXene substrate and were explored as discussed below.

$\mathrm{N}_{2}$ chemisorption was then examined to evaluate the effectiveness of this SAC system design. As shown in Fig. 1(a), since the single-atom catalysts are embedded in the original Ti vacancies, three partially exposed metallic sites will be available, namely the single-atom catalyst and the two adjacent Ti atoms that are arranged in an equilateral triangle. Considering the multiple active site geometry, two $\mathrm{N}_{2}$ adsorption configurations are possible, namely the "side-on" pattern and the "end-on" pattern, as shown in the insets of Figs. 2(a) and 2(e) respectively. For the former pattern, $\mathrm{N}_{2}$ is horizontally anchored with both sides interacting with the substrate, while for the latter, only one side of $\mathrm{N}_{2}$ coordinates with the single-atom catalyst, because of the vertical geometry. It is important to note that the dissociation of $\mathrm{N}_{2}$ is not observed, suggesting the associative reaction mechanism. The evolution of the binding strength with the number of $d$ electrons is presented in Figs. 2(a) and 2(e), and interestingly we find that for both adsorption patterns, with the increasing number of $d$ electrons, the binding energy first gradually increases and then decreases, with the energies in the range from $-2.2 \mathrm{eV}$ to $0 \mathrm{eV}$. Furthermore, for the early transitional metals, the most stable adsorption configuration is side-on, while the end-on pattern gradually becomes preferred as the number of the $d$ electrons is increased, with the switch from side-on preferred to end-on preferred occurred at the $d^{5}$ and $d^{6}$ systems of $\mathrm{Fe}, \mathrm{Ru}, \mathrm{Re}$.

The origins of the electronic structure of these materials were further explored to examine whether this design can achieve effective electronic interactions. Herein, we used Co as a representative system, and plotted the PDOS and crystal occupation Hamiltonian (COHP) for the side-on pattern as shown (a)

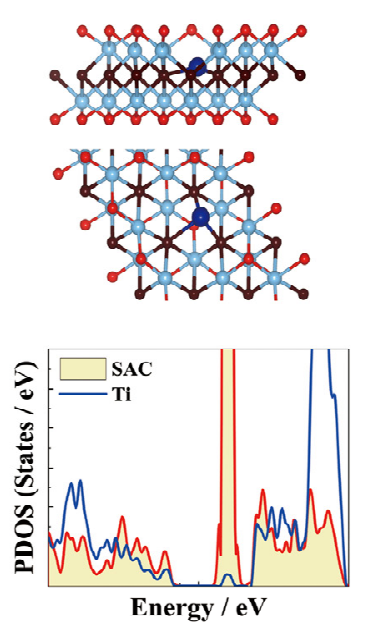

(b) -
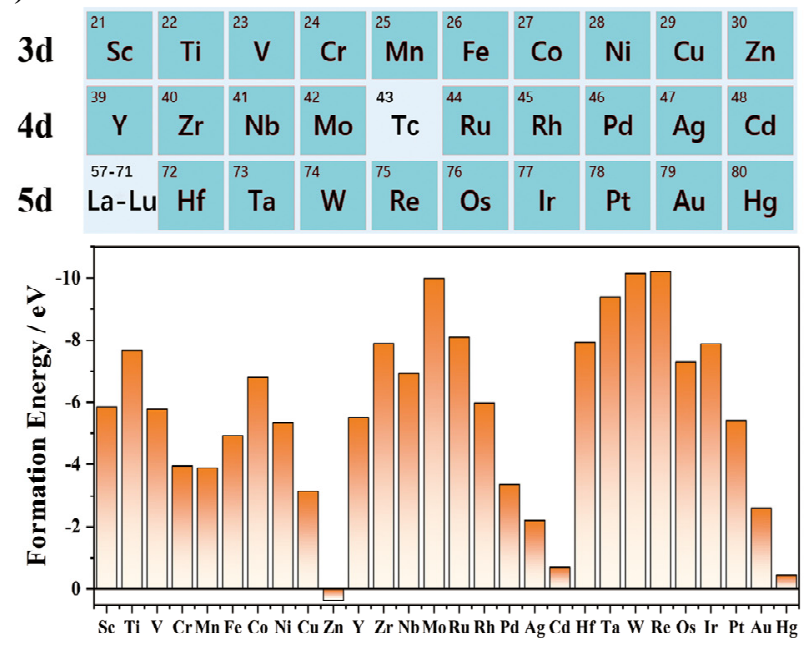

Fig. 1. (a) Side and top views of the atomic configurations of the SAC@MXene design (upper panel) and the general features of the partial density of states (lower panel); (b) 28 kinds of single-atom catalysts (cyan) considered for screening and the corresponding formation energies. 
(a)

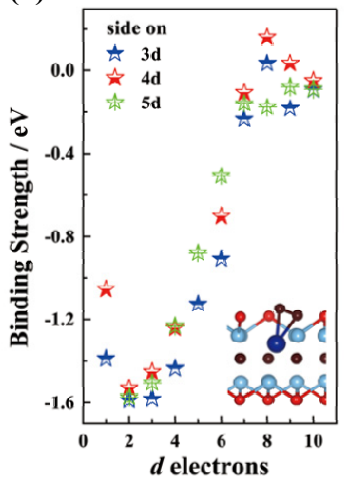

(e)

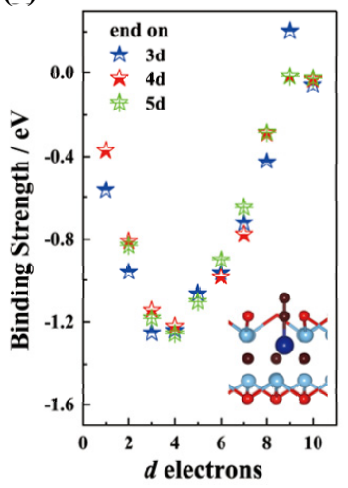

(b)

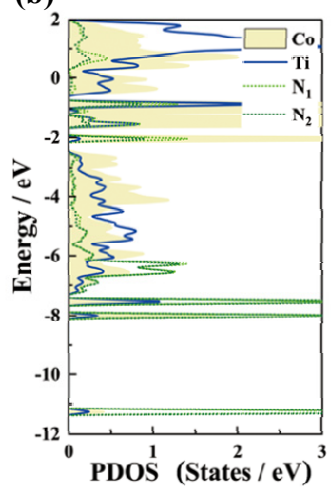

(f)

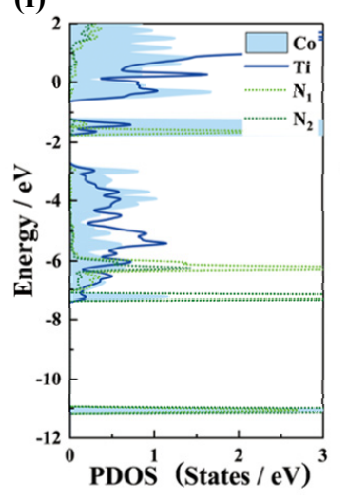

(c)

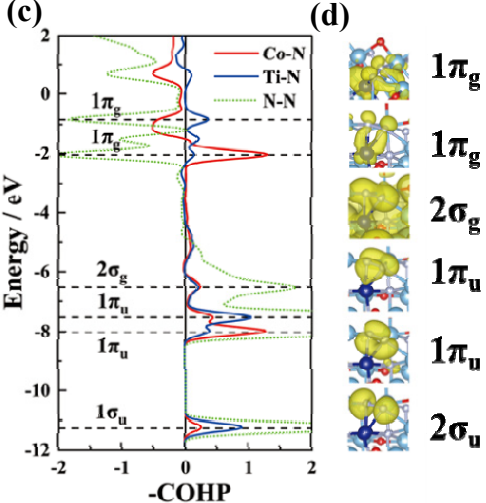

(g)

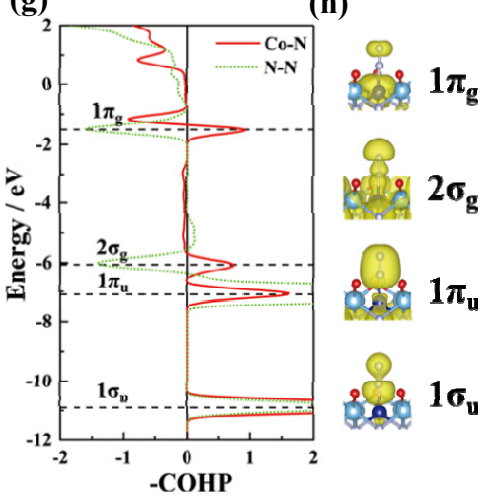

Fig. 2. $\mathrm{N}_{2}$ binding strength for the (a) side-on and (e) end-on adsorption patterns. Partial density of states (PDOS) for the (b) side-on and (f) end-on adsorption patterns. Crystal occupation Hamiltonian population (COHP) for the (c) side-on and (g) end-on adsorption patterns. Spatial charge distribution for the (d) side-on and (h) end-on adsorption patterns. The adsorption configuration is shown in the inset of the left panel.

in Figs. 2(b) and 2(c). The results indicate that the lower broadened Co states overlap with three kinds of electronic states of $\mathrm{N}_{2}$. The two sharp states located at approximately -8 $\mathrm{eV}$ can be attributed to the coordination with Co atom and with the hybridization with the Ti atom, respectively, as validated by the bonding peaks in COHP. Another hybridized state located at $\sim-6 \mathrm{eV}$ is more broadened and simultaneously forms chemical bonds with $\mathrm{Co}$ and $\mathrm{Ti}$. The spatial charge distribution analysis in these energy intervals was further performed (Fig. 2(d)), and the obtained spatial characteristics indicate that the former two states belong to the originally double degenerate $1 \pi \mathrm{u}$ molecular orbitals of $\mathrm{N}_{2}$ and the third state corresponds to the lone-pair electrons in the $2 \sigma_{\mathrm{g}}$ molecular orbital. This demonstrates that both single-atom catalysts and adjacent Ti atoms can serve as electron acceptors by forming $\sigma$ bonds with these occupied states. Furthermore, the localized single-atom catalyst states at approximately $-1 \mathrm{eV}$ are symmetrically matched with the vertical $1 \pi_{\mathrm{g}}$ states and can donate electrons into these anti-bonding states. The other $1 \pi_{\mathrm{g}}$ state that lies parallel to the substrate can hybridize with the $d$ orbitals of the two adjacent $\mathrm{Ti}$ atoms, serving as donors to activate the $\mathrm{N}_{2}$ molecule. Therefore, this multiple orbital interaction scenario is symmetry-matched and can be viewed as an "acceptor-donor" mechanism [57] that is realized by the synergistic effect between the Co and $\mathrm{Ti}$ in the substrate.

For other single-atom catalysts, this synergistic effect is also present but varies with the number of $d$ electrons. To further examine this effect, we calculated the integrated COHP (ICOHP) values to evaluate the effectiveness of this interaction (Fig. S7). The results indicate that for elements with half-full $d$-electron shell, the donor-acceptor interaction is the strongest and has the highest ICOHP value, in accordance with the binding strength trend. For the end-on pattern (inset in Fig. 2(e)), the PDOS, COHP and spatial charge distribution (Figs. 2(f), 2(g) and $2(\mathrm{~h})$, respectively) show that the lone-pair electrons in $1 \sigma_{u} / 2 \sigma_{\mathrm{g}}$ and bonding electrons in $1 \pi_{\mathrm{u}}$ can all coordinate with the Co atom that acts as an acceptor capturing electrons from the occupied states in $\mathrm{N}_{2}$. Meanwhile, the spatial distribution of the anti-bonding $1 \pi \mathrm{g}$ states is strongly changed due to its occupation by the electrons donated by the Co atom. Thus, the orbital symmetry matching and the acceptor-donor interaction are also present in the end-on pattern. Similarly, we have performed ICOHP analysis (Fig. S7) and found that the obtained ICOHP values exhibits the same trend as the binding ability shown in Fig. 2(e).

\subsection{Electrochemical performance of NRR}

Following the evaluation of the $\mathrm{N}_{2}$ adsorption ability, the electrochemical performance of NRR was examined. Generally, NRR is modeled using three typical reaction mechanisms, namely the distal, alternating, and enzymatic mechanisms [16,34]. For the end-on adsorption pattern, the two mechanisms can be divided into distal and alternating mechanisms 
(a)

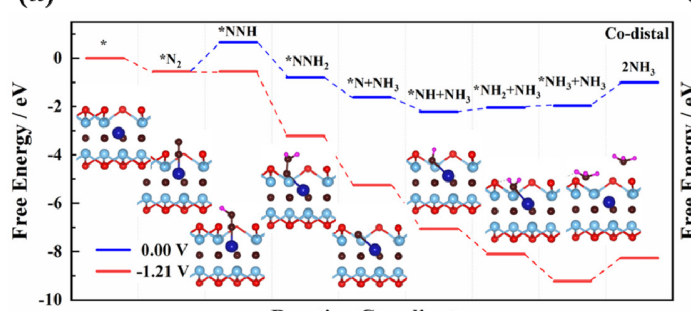

(b)

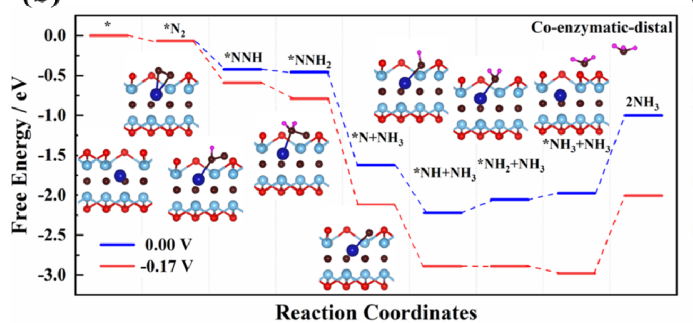

(b)

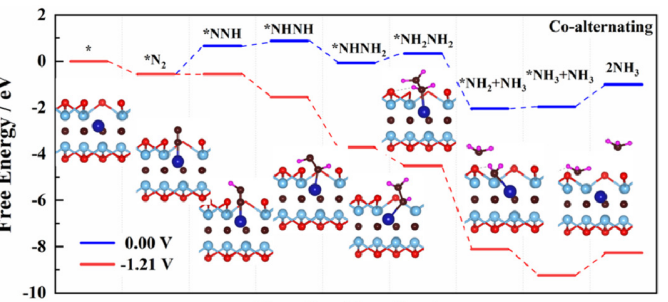

(d)

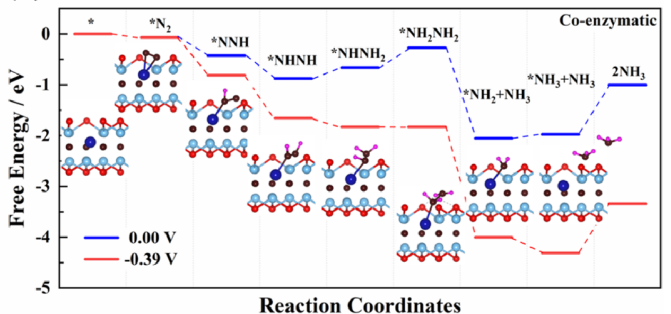

Fig. 3. Free energy evolution process for the (a) distal, (b) alternating, (c) enzymatic-distal, and (d) enzymatic reaction mechanisms, where structural evolution is shown in the top bar of each panel.

based on whether or not the attacking site of the hydrogen is preferred. As shown in the upper panel of Fig. 3(a), in the distal mechanism, hydrogen prefers to attack the distal $\mathrm{N}$, and then dissociation occurs, releasing the first $\mathrm{NH}_{3}$. For the alternating reaction mechanism, the hydrogenation sequence alternates between the two $\mathrm{N}$ atoms that will dissociate in the last step, producing two $\mathrm{NH}_{3}$ simultaneously. Meanwhile, the enzymatic mechanism starts from the side-on adsorbed configuration and proceeds through an alternating hydrogenation process, as shown in Fig. 3(d). Nevertheless, if the two $\mathrm{N}$ atoms are not symmetric, similar to the reaction for the end-on adsorption pattern in our SAC-MXene design, hydrogen may attack one side of the $\mathrm{N}_{2}{ }^{*}$ species preferentially, leading to a mixed enzymatic-distal mechanism (Fig. 3(c)) in which only one $\mathrm{N}$ atom will be hydrogenated and will dissociate at the intermediate step, with the mechanism then proceeding along the distal mechanism pathway. In the following discussion, we consider four kinds of reaction pathways for all SAC-MXene systems, and focus on the energetically favorite pathway with the lowest onset potential.

The simulated free energy evolution for Co-MXene is presented in Fig. 3, while the pathways for the other systems are shown in Section SII. Different mechanisms can induce quite different free energy pathways, potential determining steps (PDS) and thus the lowest onset potentials. For both the distal and alternating mechanisms, the free energy increases strongly in the attack of the first hydrogen, and this is the PDS for most SAC candidate systems. For the Co-MXene system, the first attached hydrogen elongates the $\mathrm{N}-\mathrm{N}$ bond length from 1.14 to $1.21 \AA$, while the Co-N bonds shorten from 1.78 to $1.68 \AA$. This process is accompanied by the uphill change in the free energy by $1.21 \mathrm{eV}$ that determines the onset potential for both the distal and alternating reaction pathways. Then, for the distal reaction shown in Fig. 3(a), the distal $\mathrm{N}$ is further attacked and the entire reaction proceeds smoothly from ${ }^{*} \mathrm{NNH}$ to ${ }^{*} \mathrm{NNH}_{2}$ with decreasing free energies, finally producing the first ammonia molecule. During this process, the end-on adsorption site will move from the top of the single-atom catalyst to the center of the Co-Ti-Ti cluster, where the subsequent hydrogenation of * $\mathrm{N}$ occurs. For the evolution of the second ammonia molecule, the free energy gradually increases from ${ }^{*} \mathrm{NH}$ to ${ }^{*} \mathrm{NH}_{2}$ and finally to ${ }^{*} \mathrm{NH}_{3}$, but with a smaller increase in the free energy. It is important to note that for the early and late single-atom catalysts such as $\mathrm{Y}, \mathrm{Nb}$, and $\mathrm{Pd}$, the distal mechanism will be mixed with one or two configurations in the enzymatic mechanism during the initial hydrogenation steps. Since these single-atom catalyst candidates possess either nearly full or nearly empty $d$ electron shells, the involvement of the adjacent Ti atoms is required to stabilize the intermediates, producing the mixed side-on configurations and eliminating the barrier for the first hydrogenation step. For the alternating mechanism, the second hydrogen prefers to attack the proximal N, distorting the vertical adsorption configuration and lengthening the Co-N bonds from 1.68 to $1.88 \AA$ Å. During this stage, free energy still increases, and such an uphill energy change can even compete with the first step as the PDS. Generally, in the alternating hydrogenation sequence, the distortion and rotation of the intermediates can be observed, resulting in a zigzag-free energy evolution profile. As a result, energy increases in three regions, namely 1) $\left.{ }^{*} \mathrm{~N}_{2} \rightarrow{ }^{*} \mathrm{NNH} \rightarrow{ }^{*} \mathrm{NHNH}, 2\right){ }^{*} \mathrm{NHNH}_{2} \rightarrow$ $* \mathrm{NH}_{2} \mathrm{NH}_{2}$, and 3) ${ }^{*} \mathrm{NH}_{2} \rightarrow * \mathrm{NH}_{3}$.

For the enzymatic-distal reaction path, the free energy gradually decreases during the production of the first ammonia molecule. Unlike for the distal and alternating reaction pathways, the first hydrogenation step is energetically favorable for the side-on pattern, while the N-N bond length changes from 1.25 to $1.34 \AA$ along with a downhill energy change by $0.36 \mathrm{eV}$ for the Co system. From ${ }^{*} \mathrm{~N}_{2}$ to ${ }^{*} \mathrm{NNH}$ and $\mathrm{NNH}_{2}$, the $\mathrm{N}-\mathrm{N}$ dimer gradually rotates its direction to change its coordination with both the single-atom catalyst and the Ti atoms, facilitating subsequent hydrogenation (Fig. S8). During the evolution of the second ammonia, the lowest free energy was achieved when forming the ${ }^{*} \mathrm{NH}$ species, and then was followed by the ${ }^{*} \mathrm{NH} \rightarrow{ }^{*} \mathrm{NH}_{2} \rightarrow{ }^{*} \mathrm{NH}_{3}$ steps that were all uphill in energy. Thus, 
the PDS is located in this region. Specifically, the step from ${ }^{*} \mathrm{NH}_{2}$ to ${ }^{*} \mathrm{NH}_{3}$ is the PDS and gives rise to a much smaller onset potential than that in the first step of the distal and alternating mechanisms. Meanwhile, for the enzymatic reaction, the side-on configuration is gradually rotated, but is always retained during the six-step hydrogenation process (Fig. S9). The PDS appears during the transition from ${ }^{*} \mathrm{NHNH}_{2}$ to ${ }^{*} \mathrm{NH}_{2} \mathrm{NH}_{2}$ and requires more energy than the PDS of the distal-enzymatic reaction pathway, leading to an onset potential of $-0.39 \mathrm{~V}$ for the Co system. An examination of all of the reaction mechanisms of all SAC systems in section SII further shows that for the side-on pattern, the enzymatic-distal mixed mechanism usually has the lowest onset potential, while only the Sc, Y, Cr catalytic systems will allow the reaction through the enzymatic mechanism. For the end-on adsorption, distal reaction is always preferred to the alternating mechanism. Therefore, we concentrate on the distal and enzymatic-distal mechanisms in the discussions in the next section.

The hydrogen evolution reaction (HER) is the major competitive reaction for NRR that consumes protons and electrons [58] and is even preferred to the NRR process for pure transition metals [59]. Therefore, we simulate the HER performance here. The common three-step evolution process with the initial $\mathrm{H}^{+}$state, intermediate $\mathrm{H}^{*}$, and final $1 / 2 \mathrm{H}_{2}$ product was adopted [60]. The free energy evolution and adsorption configurations for the $3 d$ elements are presented in Fig. 4(a), while the results for the others elements are shown in Fig. S10. An examination of Fig. 4(a) indicates that hydrogen will simultaneously bond with the single-atom catalyst and the adjacent Ti atoms, leading to high binding strength. Since a Gibbs free energy difference close to zero is the prerequisite for an ideal HER catalysis, the designed SAC-MXene will strongly hinder the HER process. Fig. 4(b) further shows a comparison of the overall NRR and HER performance, where the dashed line denotes that the two processes have identical onset potentials. The results indicate that for all considered candidate systems, NRR performance always surpasses that of the HER process.

\subsection{Electronic structure origins of the rate determining step and linear scaling relations}

The effectiveness of the catalysts for $\mathrm{N}_{2}$ was verified as de- scribed above in the first section, due to the symmetrically matched states that are a general feature in this SAC@MXene design. However, the PDS is another aspect that controls the electrochemical performance. In the traditional BEP relationship, the dissociation of $\mathrm{N}_{2}$ in the first step faces a sizable energy barrier that shows a contradictory relationship with the bonds to the substrate to the barrier for the desorption of $* \mathrm{NH}_{3}$, thus limiting the lowest onset potential $[7,8]$. By contrast, for the SAC-MXene design, based on maintaining the orbital matched side-on/end-on pattern, it is possible to break this restriction.

In the enzymatic-distal mechanism, PDS is usually found in the transition from ${ }^{*} \mathrm{NH}_{2}$ to $\mathrm{NH}_{3}$, and therefore, the electronic structure of the ${ }^{*} \mathrm{NH}_{2}$ adsorption system was examined. Taking Co system as a typical example, the partial density of states, the results of COHP analysis and the spatial charge distribution are shown in Fig. 5(a). The deep lying states $\sim-8.5 \mathrm{eV}$ correspond to the strong $\sigma \mathrm{N}-\mathrm{H}$ bonds, while the broadened states observed in the range from -8 to $-2 \mathrm{eV}$ can be mainly attributed to Co-N bonds. The spatial charge distribution in this energy interval verifies that both lone-pair electron and the unbonded $s p^{3}$ electron in $\mathrm{N}$ atom can coordinate with the single-atom catalyst, in accordance with the two bonding peaks in the COHP. When the final hydrogen attacks, this SAC-N interaction is disrupted and a new $\sigma \mathrm{N}-\mathrm{H}$ bond forms, leading to the uphill change in the energy. Therefore, it can be expected that by weakening this SAC-N bond and thus the adsorption ability toward ${ }^{*} \mathrm{~N},{ }^{*} \mathrm{NH}_{2}$ and ${ }^{*} \mathrm{NH}_{3}$ species, a lower onset potential can be achieved, while the initial coordination between $\mathrm{N}_{2}$ and the SAC-MXene can still be maintained due to the symmetry-matched geometry. Therefore, using the binding strength of a single $\mathrm{N}$ atom $\left(E_{\text {single-N }}\right)$ as an measure of the adsorption ability, the evolution of the onset potential in the enzymatic-distal mechanism is plotted in Fig. 5(b). A linear scaling relation is observed with lower $E_{\text {single-N }}$ corresponding to higher onset potential. The optimal performance is obtained in the $d^{8}$ electron systems such as $\mathrm{Ni}, \mathrm{Pd}, \mathrm{Pt}$, with the onset potentials as low as $-0.13,-0.24$ and $-0.29 \mathrm{~V}$, respectively.

For the end-on pattern with the distal/alternating mechanism, the PDS is generally the first hydrogenation process, while the uphill change from ${ }^{*} \mathrm{NH}_{2}$ to ${ }^{*} \mathrm{NH}_{3}$ is still present but is much less significant. The electronic structure of the ${ }^{*} \mathrm{NNH}$ (a)

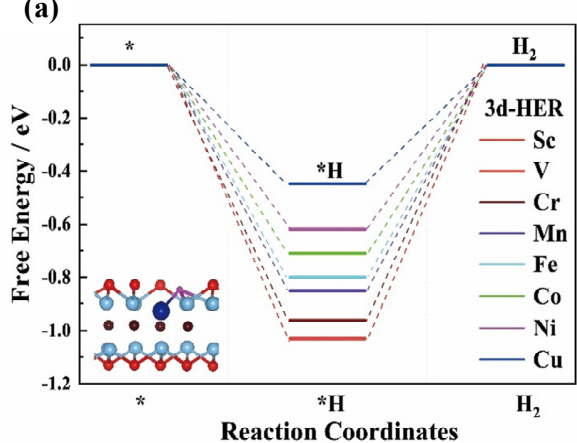

(b)

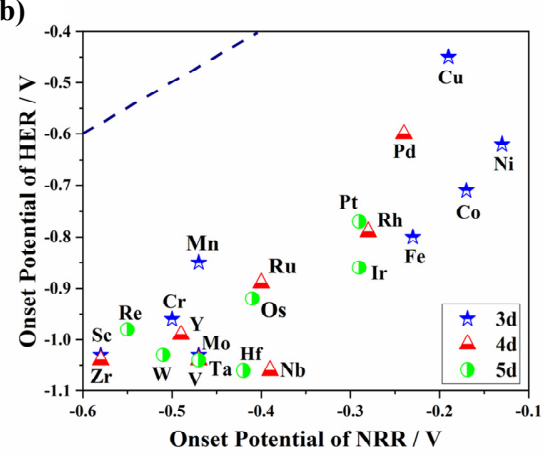

Fig. 4. (a) Free energy evolution for the HER process, where the H adsorption configuration is shown in the inset. (b) Comparison of the electrochemical performances of the NRR and HER processes. The dashed line represents equal onset potentials for HER and NRR, while for the elements below this line, NRR is preferred to HER. 
(a)
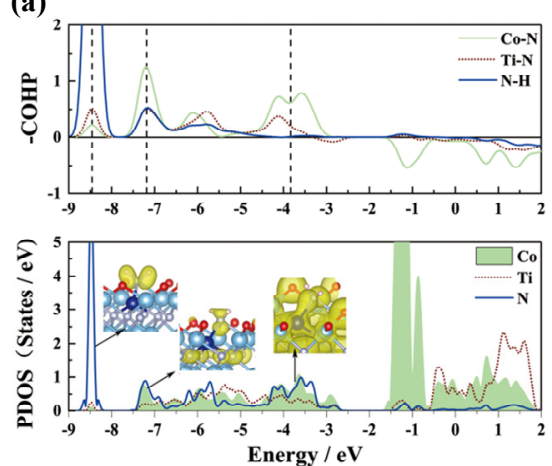

(c)

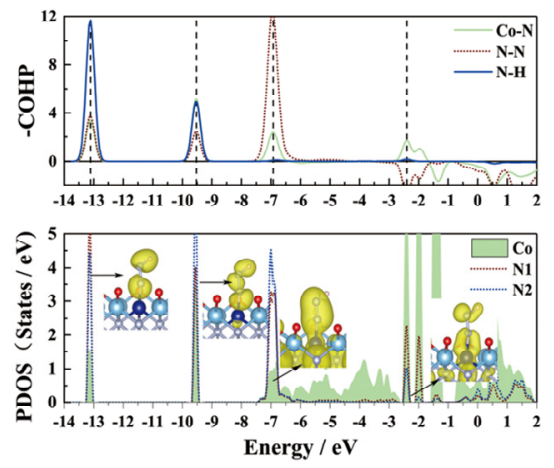

(b)

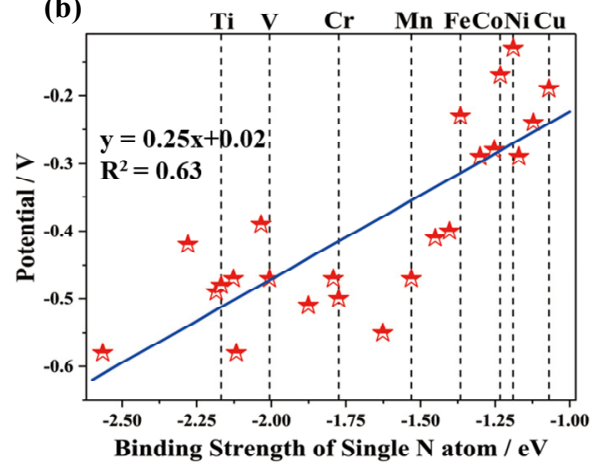

(d)

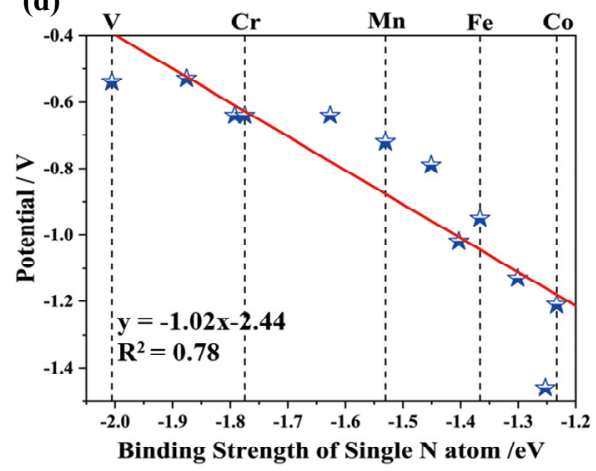

Fig. 5. COHP and partial density of states for the (a) ${ }^{*} \mathrm{NH}_{2}$ adsorption system in the enzymatic-distal reaction path and (c) ${ }^{*} \mathrm{NNH}$ adsorption system in the distal reaction path, where the spatial charge distribution in each energy interval is shown in the inset with the isosurface set to $0.008 \mathrm{e} / \AA^{3}$. The scaling relations between the onset potential and the binding strength of a single $\mathrm{N}$ atom for the (b) enzymatic-distal reaction and (d) distal reaction.

adsorbed system is presented in Fig. 5(c). It is observed that the strongest $\mathrm{N}-\mathrm{H}$ bond overlaps with the lower lying $1 \sigma_{\mathrm{u}}$ states at $\sim-13 \mathrm{eV}$ along with Co-N bonding states in this energy region. After the first step, the peak intensity of the Co-N bond is greatly weakened, indicating that the original SAC- $\mathrm{N}_{2}$ interaction is sacrificed in the first step. Thus, the enhanced SAC- $\mathrm{N}_{2}$ strength can promote the ${ }^{*} \mathrm{~N}_{2}$ to acquire electrons from hydrogen more effectively, further mediating the onset potential. To verify this analysis, the binding strength of a single $\mathrm{N}$ atom is also used here as a descriptor of the SAC- $\mathrm{N}_{2}$ interaction, and a similar linear relation with the onset potential is also established in Fig. 5(d) for the distal reaction, demonstrating that higher binding strength corresponds to lower onset potential (while some elements adopt mixed distal reaction pathway, eliminating such first step PDS, and are not included). It is important to note that the increasing binding strength will lead to a side effect during the desorption of $\mathrm{NH}_{x}$ species. Therefore, the uphill free energy change in the step from ${ }^{*} \mathrm{NH}_{2}$ to ${ }^{*} \mathrm{NH}_{3}$ will be increased, but this energy barrier still cannot compete with the barrier for the first hydrogenation .

\section{Conclusions}

To conclude, we have systematically explored the electrochemical NRR performance for 28 kinds of single-atom catalysts embedded in a MXene. Formation energy and AIMD simulation results reveal that MXene is a promising substrate that can effectively accommodate SAC. Further investigation of $\mathrm{N}_{2}$ adsorption showed that the synergistic effects exist between the singe-atom catalyst and $\mathrm{Ti}$, leading to orbital symmetry matching with the inert dinitrogen and further realizing the "donor-acceptor" interaction scenario. Then, the electrochemical performance was evaluated for four different reaction mechanisms, namely the distal, alternating, enzymatic-distal, and enzymatic mechanisms that are all preferred relative to the HER. It was further discovered that SAC@MXene systems generally allow the enzymatic-distal reaction with the lowest onset potential, and the electronic structure origins of the potential determining step were elaborated. Based on this, the scaling relationship between the single $\mathrm{N}$ atom binding strength and onset potential can be established, verifying that excellent NRR performance can be achieved by using $\mathrm{Ni}$ as the SAC with the onset potential as low as $-0.13 \mathrm{~V}$. This work not only explores the NRR performance of a novel SAC@MXene catalytic system, but also emphasizes the significance of orbital symmetry matching, providing theoretical guidance for the future design of NRR catalysts.

\section{References}

[1] J. Postgate, Nitrogen Fixation, 3rd ed., Cambridge University Press, New York, 1998.

[2] J. W. Erisman, M. A. Sutton, J. Galloway, Z. Klimont, W. Winiwarter, Nat. Geosci., 2008, 1, 636-639.

[3] S. Giddey, S. P. S. Badwal, A. Kulkarni, Int. J. Hydrogen Energy, 2013, 38, 14576-14594. 


\section{Graphical Abstract}

Chin. J. Catal., 2021, 42: 288-296 doi: 10.1016/S1872-2067(20)63643-9

Orbital symmetry matching: Achieving superior nitrogen reduction reaction over single-atom catalysts anchored on Mxene substrates

Jiale Qu, Jiewen Xiao, Hetian Chen, Xiaopeng Liu, Tianshuai Wang, Qianfan Zhang*

Beihang University

Single atom doped on MXene, which can synergize with the adjacent titanium atom on the matrix. With this synergy, the nitrogen reduction reaction can be proceeded at a low limiting potential.

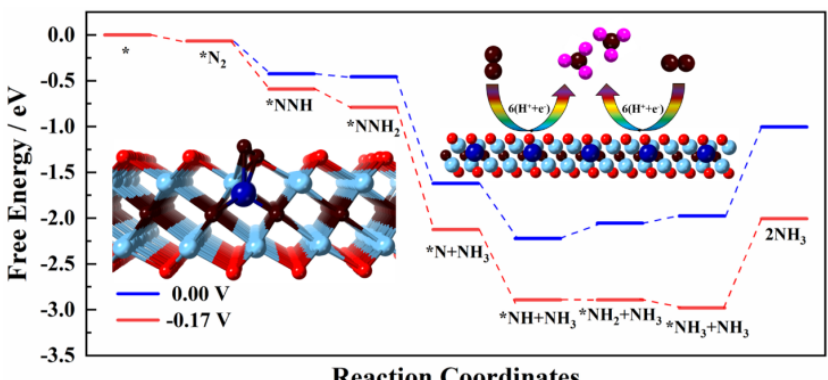

Reaction Coordinates
[4] I. A. Amar, R. Lan, C. T. G. Petit, S. J. Tao, Solid State Electrochem., 2011, 15, 1845-1860.

[5] V. Smil, Nature, 1999, 400, 415.

[6] V. Smil, Sci Am., 1997, 277, 76-81.

[7] A. J. Medford, A. Vojvodic, J. S. Hummelshøj, J. Voss, F. Ablid-Pedersen, F. Studt, T. Bligaard, A. Nilsson, J. K. Nørskov, J. Catal., 2015, 328, 36-42.

[8] T. Bligaard, J. K. Nørskov, S. Dahl, J. Matthiesen, C. H. Christensen, J. Sehested, J. Catal., 2004, 224, 206-217.

[9] Y. Nishibayashi, Inorg. Chem., 2015, 54, 9234-9247.

[10] C. J. M. Van der Ham, M. T. M. Koper, D. G. H. Hetterscheid, Chem. Soc. Rev., 2014, 43, 5183-5191.

[11] X. Cui, C. Tang, Q. Zhang, Adv. Energy Mater., 2018, 8, 1800369.

[12] X. H. Guo, Y. P. Zhu, T. Y. Ma, J. Energy Chem., 2017, 26, 1107-1116.

[13] H. P. Jia, E. A. Quadrelli, Chem. Soc. Rev., 2014, 43, 547-564.

[14] S. Kuriyama, K. Arashiba, K. Nakajima, Y. Matsuo, H. Tanaka, K. Ishii, K. Yoshizawa, Y. Nishibayashi, Nat. Commun., 2016, 7, 12181.

[15] S. Kuriyama, K. Arashiba, H. Tanaka, Y. Matsuo, K. Nakajima, K. Yoshizawa, Y. Nishibayashi, Angew. Chem. Int. Ed., 2016, 55, 14291-14295.

[16] J. Zhao, Z. Chen, J. Am. Chem. Soc., 2017, 139, 12480-12487.

[17] X. Liu, Y. Jiao, Y. Zheng, M. Jaroniec, S. Z. Qian, J. Am. Chem. Soc., 2019, 141, 9664-9672.

[18] J. Nash, X. Yang, J. Anibal, J. H. Wang, Y. S. Yan, B. Xu, J. Electrochem. Soc., 2017, 164, F1712-F1716.

[19] Y. Yao, S. Zhu, H. Wang, H. Li, M. Shao, J. Am. Chem. Soc., 2018, 140, 1496-1501.

[20] D. Wang, L. M. Azofra, M. Harb, L. Cavallo, X. Zhang, B. H. R. Suryanto, D. R. MacFarlane, ChemSucChem, 2018, 11, 3416-3422.

[21] E. Skulason, T. Bligaard, S. Gudmundsdottir, F. Studt, J. Rossmeisl, F. Abild-Pedersen, T. Vegge, H. Jonsson, J. K. Norskøv, Phys. Chem. Phys. Chem., 2012, 14, 1235-1245.

[22] A. Wang, J. Li, T. Zhang, Nat. Rev. Chem., 2018, 2, 65-81.

[23] Y. Gao, H. Zhou, Y Cao, X. Sun, G. Zhuang, S. Deng, X. Zhong, Z. Wei, J. Wang, Chin. J. Catal., 2019, 40, 152-159.

[24] B. Qiao, A. Wang, X. Yang, L. F. Allard, Z. Jiang, Y. Cui, J. Liu, J. Li, T. Zhang, Nat. Chem., 2011, 3, 634-641.

[25] B. Qiao, J. Liu, Y. Wang, Q. Lin, X. Liu, A. Wang, J. Li, T. Zhang, J. J. Liu, ACS Catal., 2015, 5, 6249-6254.

[26] B. Qiao, J. X. Liang, A. Wang, C.Q. Xu, J. Li, T. Zhang, J. J. Liu, Nano Res., 2015, 8, 2913-2924.
[27] J. X. Liang, J. Lin, X. F. Yang, A. Wang, B. Qiao, J. Liu, T. Zhang, J. Li, J. Phys. Chem. C, 2014, 118, 21945-21951.

[28] D. A. Bulushev. M. Zacharska, A. S. Lisitsyn, Q. Y. Podyacheva, F. S. Hage, Q. M. Ramasse, U. Bangert, L. G. Bulusheva, ACS Catal., 2016, 6, 3442-3451.

[29] G. Vile, D. Albani, M. Nachtegaal, Z. Chen, D. Dontsova, M. Antonietti, N. Lopez, J. Perez-Ramirez, Angew. Chem. Int. Ed., 2015, 54, 11265-11269.

[30] H. Zhang, L. Yu, T. Chen. W. Zhou, X. W. Lou, Adv. Funct. Mater., 2018, 28, 1807086.

[31] S. J. Wei, A. Li, J. C. Liu, Z. Li, W. X. Chen, Y. Gong, Q. H. Zhang, W. C. Cheong, Y. Wang, L. R. Zheng, H. Xiao, C. Chen, D. C. Wang, Q. Peng, L. Gu, X. D. Han, J. Li, Y. D. Li, Nat. Nanotechnol, 2018, 13, 856-861.

[32] H. Tao, C. Choi, L. X. Ding, Z. Jiang, Z. Han, M. Jia, Q. Fan, Y. Gao, H. Wang, A. W. Robertson, S. Hong, Y. Jung, S. Liu, Z. Sun, Chem, 2019, 5, 204-214.

[33] M. M. Shi, D. Bao, B. R. Wulan, Y. H. Li, Y. F. Zhang, J. M. Yan, Q. Jiang, Adv. Mater., 2017, 29, 1606550.

[34] C. Ling, X. Niu, Q. Li, A. Du, J. Wang, J. Am. Chem. Soc., 2018, 140, 14161-14168.

[35] M. Naguib, O. Mashtalir, Y. Carle, V. Presser, J. Lu, L. Hultman, Y. Gogotsi, M. W. Barsoum, ACS Nano, 2012, 6, 1322-1331.

[36] M. Naguib, M. Kurtoglu, V. Presser, J. Lu, J. Niu, M. Heon, L. Hultman, Y. Gogotsi, M. W. Barsoum, Adv. Mater., 2011, 23, 4248-4253.

[37] X. Liang, A. Garsuch, L. F. Nazar, Angew, Chem. Int. Ed., 2015, 54, 3907-3911.

[38] D. Er, J. Li, M. Naguib, Y. Gogotsi, V. B. Shenoy, ACS Appl. Mater. Interfaces, 2014, 6, 11173-11179.

[39] W. F. Chen, C. H. Wang, K. Sasaki, N. Marinkovic, W. Xu, J. T. Muckerman, T. Zhu, R. R. Adzic, Energy Environ. Sci., 2013, 6, 943-951.

[40] A. D. Handoko, K. H. Khoo, T. L. Tan, H. M. Jin, Z. W. Seh, J. Mater. Chem. A, 2018, 6, 21885-21890.

[41] L. M. Azofra, N. Li, D. R. MacFarlane, C. Sun, Energy Environ. Sci,, 2016, 9, 2545-2549.

[42] Z. Li, Y. R. Cui, Z. W. Wu, C. Milligan, L. Zhou, G. Mitchell, B. Xu, E. Shi, J. T. Miller, F. H. Ribeiro, Y. Wu, Nat. Catal., 2018, 1, 349-355.

[43] D. Zhao, Z. Chen, W. Yang, S. Liu, X. Zhang, Y. Yu, W. C. Cheong, L. Zheng, F. Ren, G. Ying, X. Cao, D. Wang, Q. Peng, G. Wang, C. Chen, J. Am. Chem. Soc., 2019, 141, 4086-4093.

[44] G. Kresse, J. Furthmuller, Comput. Mater. Sci., 1996, 6, 15-50.

[45] G. Kresse, J. Furthmuller, Phys. Rev. B, 1996, 54, 11169-11186. 
[46] P. E. Blochl, Phys. Rev. B, 1994, 50, 17953-17979.

[47] G. Kresse, D. Joubert, Phys. Rev. B, 1999, 59, 1758.

[48] J. P. Perdew, K. Burke, M. Ernzerhof, Phys. Rev. Lett., 1996, 77, 3865-3868.

[49] H. J. Monkhorst, J. D. Pack, Phys. Rev. B, 1976, 13, 5188-5192.

[50] R. Dronskowski, P. E. Blochl, J. Phys. Chem., 1993, 97, 8617-8624.

[51] V. L. Deringer, A. L. Tchougreeff, R. Dronskowski, J. Phys. Chem. A, 2011, 115, 5461-5466.

[52] S. Maintz, V. L. Deringer, A. L. Tchougreeff, R. Dronskowski, J. Comput. Chem., 2013, 34, 2557-2567.

[53] S. Maintz, V. L. Deringer, A. L. Tchougreeff, R. Dronskowski, J.
Comput. Chem., 2016, 37, 1030-1035.

[54] G. Kresse, J. Hafner, Phys. Rev B, 1993, 47, 558-561.

[55] G. Kresse, J. Hafner, Phys. Rev. B, 1994, 49, 14251-14269.

[56] J. K. Nørskov, T. Bligaard, A. Logadottir, J. R. Kitchin, J. G. Chen, S. Pandelov, U. Stimming, J. Electrochem. Soc., 2005, 152, J23-J26.

[57] W. Zhang, W. Zheng, Adv. Funct. Mater., 2016, 26, 2988-2993.

[58] C. Choi, S. Back, N. Kim, J. Lim, Y. Kim, Y. Jung, ACS Catal., 2018, 8, 7517-7525.

[59] J. H. Montoya, C. Tsai, A. Vojvodic, J. K. Nørskov, ChemSusChem, 2015, 8, 2180-2186.

[60] Q. Tang, D. Jiang, ACS Catal., 2016, 6, 4953-4961.

\title{
轨道对称性匹配: MXene基体上的单原子催化以实现优异的氮还原反应
}

\author{
曲家乐, 肖杰文, 陈和田, 刘晓鹏, 王天帅, 张千帆* \\ 北京航空航天大学材料科学与工程学院, 北京100191
}

\begin{abstract}
摘要: 氨作为一种可被植物直接吸收用以合成其他有机物的重要成分, 在化学化工及含氮化合物的生产当中起着至关重 要的作用. 传统工业生产氨气采用Haber-Bosch工艺, 将空气中丰富的氮气转化为氨气, 但该工艺需要较高的压强和温度来 促进氮气分解, 因此会消耗大量能源. 近年来, 电催化反应发展迅速. 在电催化工艺中, 通过控制操作电位及电解质便可提 高生产效率, 降低能源消耗. 基于这种策略, 各种针对能源环境的催化研究应运而生, 如二氧化碳还原、水分解反应等. 其 中, 对于氮还原的催化研究尤其是电催化设计领域研究相对较少. 研究发现, 在电催化剂中, 异构掺杂及原子尺度的调控 可以极大地影响催化剂的催化活性. 其中, 单原子催化(SAC)因其在催化活性和催化选择性上的优势受到广泛关注. MXene是一种二维过渡金属碳化物或氮化物, 其优异的化学性能和稳定的表面构型可以对单原子起到良好的针定与支撑 作用, 是一种更具潜力的单原子催化基体. 本文基于上述思想, 利用密度泛函第一性原理等模拟软件, 设计并研究了以 MXene为基体的28种过渡金属单原子催化体系, 计算并分析了各SAC@MXene体系对氮还原反应的催化效果, 从限制电 势、催化路径、反应机制等方面探索了其催化性能. 并对体系进行了态密度、晶体轨道哈密顿量、差分电荷密度等电子 结构分析, 找到了适用于MXene体系的单原子催化设计原则.

通过对限制电势的计算表明, Ni@MXene和Co@MXene体系具有很低的限制电势 $(-0.13$ 和 $-0.17 \mathrm{~V})$, 说明这些体系在 较低的启动电压下即可发生氮还原反应. 研究发现了一种新型适用于SAC@MXene氮催化体系的酶促-远端反应机制. 电 子结构分析得到 SAC原子与MXene基体的 $\mathrm{Ti}$ 原子在催化过程中存在一种协同作用. 态密度及晶体轨道哈密顿量也显示出 SAC原子与MXene基体 Ti之间的一种轨道对称性匹配关系, 揭示了这种协同作用对催化反应的积极作用. 计算的氢析出反 应(HER)结果也显示, 在相同化学环境下, SAC@MXene体系氮还原反应相对于氢析出反应更易发生.
\end{abstract}

关键词: 轨道对称性匹配; 单原子催化剂; 氮还原反应; MXene基体; 电位决速步

收稿日期: 2020-03-17. 接受日期: 2020-04-30. 出版日期: 2021-02-05.

*通讯联系人. 电话: 15210659892; 传真: (010)82314478; 电子信箱: qianfan@buaa.edu.cn

基金来源: 国家自然科学基金(11404017).

本文的电子版全文由Elsevier出版社在ScienceDirect上出版(http://www.sciencedirect.com/science/journal/18722067). 